\title{
Ways of Knowing When Research Subjects Care
}

\author{
Dorothy Howard \\ Communication \& Design Lab \\ San Diego, CA \\ dhoward@ucsd.edu
}

\author{
Lilly Irani \\ Communication \& Design Lab \\ San Diego, CA \\ lirani@ucsd.edu
}

\begin{abstract}
This paper investigates a hidden dimension of research with real world stakes: research subjects who care - sometimes deeply - about the topic of the research in which they participate. They manifest this care, we show, by managing how they are represented in the research process, by exercising politics in shaping knowledge production, and sometimes in experiencing trauma in the process. We draw first-hand reflections on participation in diversity research on Wikipedia, transforming participants from objects of study to active negotiators of research process. We depict how care, vulnerability, harm, and emotions shape ethnographic and qualitative data. We argue that, especially in reflexive cultures, research subjects are active agents with agendas, accountabilities, and political projects of their own. We propose ethics of care and collaboration to open up new possibilities for knowledge production and socio-technical intervention in $\mathrm{HCI}$.
\end{abstract}

\section{CCS CONCEPTS}

\section{- Human-centered computing $\rightarrow$ Collaborative and so- cial computing;}

\section{KEYWORDS}

Qualitative Methods, Online Communities, Ethics, Care, Feminism, Gender

\section{ACM Reference Format:}

Dorothy Howard and Lilly Irani. 2019. Ways of Knowing When Research Subjects Care. In CHI Conference on Human Factors in Computing Systems Proceedings (CHI 2019), May 4-9, 2019, Glasgow, Scotland UK. ACM, New York, NY, USA, 16 pages. https://doi.org/ 10.1145/3290605.3300327

Permission to make digital or hard copies of all or part of this work for personal or classroom use is granted without fee provided that copies are not made or distributed for profit or commercial advantage and that copies bear this notice and the full citation on the first page. Copyrights for components of this work owned by others than the author(s) must be honored. Abstracting with credit is permitted. To copy otherwise, or republish, to post on servers or to redistribute to lists, requires prior specific permission and/or a fee. Request permissions from permissions@acm.org. CHI 2019, May 4-9, 2019, Glasgow, Scotland UK

(C) 2019 Copyright held by the owner/author(s). Publication rights licensed to ACM.

ACM ISBN 978-1-4503-5970-2/19/05...\$15.00

https://doi.org/10.1145/3290605.3300327

\section{INTRODUCTION}

Research subjects rarely have the chance to reflect on their experiences of participating in research in the worlds where researchers discuss, debate, and reflect. The voices and reflections of researchers, on the other hand, are common. Yet subjects of research certainly have situated knowledge - knowledge born of their practices and social positionality [70]. They also have their own projects - projects in which researchers may be an instrument.

There are many reasons why participants in human-computer interaction (HCI) research may have interests, needs, and desires beyond offering themselves as a repository of experience or a specimen of practice. People might participate because they are passionate or curious about technological futures and want to explore how those futures get made; this may interest them more than the particular domestic, work, or social technologies of any given research study or experiment. They may care about a social media platform such as Facebook and see research participation as an opportunity to advocate for their own needs or network with technology professionals. Anthropologists have recognized the way research can function not only as an encounter or an opportunity to observe, but as a form of collaboration that transforms researcher and researched in the process [121]. What researchers produce may not be all that is produced in the researcher-subject encounter.

The first author was involved in Wikipedia for years as an editor, as a event organizer, and as someone who worked on diversity. In this position, she, like many others, became deeply familiar with waves of scholarly research into gender dynamics in Wikipedia. She participated in not one, but ten interviews that positioned her as an informant on Wikipedia editors' experiences. Far from catharsis, she found herself laboring to support researchers, to explain sometimes painful experiences, and to speak about what she had learned while respecting that some stories were not hers to tell. This paper is a meditation on the collaborative and ethical possibilities of qualitative research, research which informs the policy, design, and maintenance of high stakes, real world systems. Our case study is Wikipedia.

HCI researchers have previously explored the ways that research methodologies shape ethics. Ethics are modes of making decisions, and they are sometimes applied to understand how research impacts various actors and institutions 
at different predictable stages in the research process like informed consent, and in situ, as sometimes unpredictable scenarios arise $[19,24]$. In this paper, we examine HCI research methods and ethics in studies that involve everyday people in the production of knowledge - human subjects in ethics broad parlance. But we do more than this; we explore how people assert themselves as knowing subjects in the research process and attempt to disentangle how research subjects manage accountability and representation, as well as the emotional labor that they may be asked to do. We find that research participants have an essential and important role in managing ethical accountabilities. These accountabilities are also hardly static, but socially constructed during research encounters as the roles of investigators and participants are negotiated. The motivations people have for participating in qualitative studies work at multiple scales, including the personal, interpersonal, and political - as research disperses through different publics in the short and long-term [34, 113]. We build on accounts of research that understand it as ethical and political - in the knowledge it produces [82, 135], in the relations it creates or reinforces [109], and in the politics it can make possible [70].

We offer an extended reflection on the experiences of one participant, the first author, who became a frequent contributor to research on gender and Wikipedia, and use this grounded experience to open up questions about common practices by which we engage research subjects - practices we teach, we often take for granted, and to which we have been habituated. We examine research as a process of coming to know the world - a world we inhabit as well - but examine the kinds of subjectivities, social relations, and possibilities for collaboration enabled or foreclosed by common qualitative research practices [135]. By subjectivity, we mean how people interpret, act, and make sense of the world using the tools they carry from prior experiences and cultural backgrounds.

This paper's focus on the experiences of participants in Wikipedia research produces insight into how people generate knowledge about platforms and practices in which they are deeply invested. These investments might be technical, social, or emotional. Such practices might include social media use, online community participation, or participation in making and design.

We extend these reflections to imagine how other research processes might be possible when we can treat research subjects as potential collaborators rather than informants. An overarching concern of this project is to revisit and foster sensitivities to the experiences of research subjects to better understand how HCI researchers can practice care and to contribute to work in $\mathrm{HCI}$ on reflexive practices.

\section{RELATED WORK: ETHICS, VALUES, POLITICS, AND CARE IN HCI}

\section{Ethics}

Very broadly, ethics primarily concerns the relationship between intentions and consequences of ones' actions. In philosophy, for example, ethics can involve evaluating what is good according to different criteria. Within HCI, calls for ethics can include ethics of research practice, as well as ethical evaluations of particular technologies such as AI, health activity trackers, or product design [19, 56, 65, 110]. Bruckman identifies a few recurrent ethical principles in $\mathrm{HCI}$ research: obtaining informed consent, 'do no harm', privacy, and the distribution of risks, costs, and benefits [24].

As technologies rework terrains of social interactions, researchers must consider possible risks, harms, and consequences for research subjects. Search engines or anonymized open data sets might still enable committed searchers to discover subject identities in ways not possible two decades ago [114, 129]. Chatrooms and forums offer visibility, but researchers cannot always rely on precedent to determine what constitutes public versus private spaces in networked settings, or when informed consent is necessary[81]. Questions of ethics often focus on dilemmas researchers face acting to reduce potential harm to subjects of research or implicated communities. Many have converged on how institutional review boards (IRB) procedures and formal guidelines can manage procedural ethics, including potential risks and harm, amid emerging digital and socio-technical research settings [25].

Works drawing on feminist, postcolonial, and decolonial approaches emphasize that these judgments and dilemmas are produced in part by the ways researchers and participants relate to one another, often differentially privileged by power structures, institutions, identities, and histories of coloniality $[82,113]$. This paper takes up this concern for ethics amidst power relations between researcher and subject. However, our contribution is to demonstrate ethical dilemmas from the position of a research subject.

\section{Values}

Another way HCI practitioners have evaluated social impact and ethics is in terms of human values [28, 85]. In a cross-disciplinary study on how 'values' are characterized and used to predict the behavior of individuals and organizations, Cheng and Fleischmann arrive at the definition that, "values serve as guiding principles of what people consider important in life" [58,59]. Their method of conceptual investigation calls for researchers to draw on "all values of moral import", including the moral, social, religious, and professional. Values, in this sense, are paradigms composed of criteria used to evaluate how different decisions can uphold 
those values. Not all values are moral ones. Friedman argues that the value of usability, for example, is not necessarily a moral as it can also lead to an ease of using dangerous objects.

Value sensitive design offers a rich method by which researchers can mediate different values among stakeholders in technologically designed futures. Methods might include ethnographic, survey, and interview work asking participants about their values and moral conceptions [35]. It offers a way to reveal tensions in values held by different participants rather than covering them over. However, stabilized methodologies do not travel universally. Borning and Muller have suggested that VSD can 'overclaim' the universality of values, but miss persistent power hierarchies between researchers and subjects [19]. Le Dantec and Poole suggest that VSD ought to discover values held by indirect and direct stakeholders rather than drawing from hegemonic literatures and philosophies [98].

The process itself also may not travel. Cultures of production, including Wikipedia's, have norms and practices that implicitly enact a set of values but require that participants engage value tensions in situ, engaging in the practice repertoires of that community. Reagle, for example, describes the fundamental Wikipedia principle of "assume good faith" (AGF), invoked when evaluating changes to the encyclopedia and navigating relationships among editors, as a cultural value produced by a community of practice $[120,143]$. The methods of VSD, then, may not be recognized as legitimate modes of governance by all communities. Struggles for recognition, inclusion, or even value shifts might require participants to negotiate methods and engage in practices of politics.

\section{Politics}

While ethics and values emphasize the individual, politics draws attention to how the collective governs. HCI researchers often write of politics as the process by which design is affected by efforts of social justice, advocacy, activism, or civic engagement. Varied theories of politics produce different understandings of the relationship of $\mathrm{HCI}$ - infrastructures, designs, and expertise about technology - to politics. Participatory Design traditions in Scandinavia engaged trade unions as way of holding technological design processes accountable to political processes of Swedish social democracy $[14,90,126]$. Compared to notions of stakeholders, politics imply the recognition of the importance of conflict and deliberation in collective life. Extending participatory design traditions, LeDantec and DiSalvo highlight how design practice can do more than create useful objects [36]. It can create infrastructures that allow communities to gather and form around shared issues and concerns. Participatory design research can further engage with communities in the coproduction of knowledge, extending the research encounter as an opportunity for communities to co-design, organize, and enact politics [108].

Pluralistic agonism, another theory of politics, posits the value of conflict between strong opponents in strengthening democracy. Drawing on this theory, DiSalvo argues that HCI might affect politics by creating informational and aesthetic designs that provoke ideological conflict [43]. Feminist $\mathrm{HCI}$ by contrast, proposes feminist values as a basis for HCI practice that promotes feminism in public life [8, 22, 123]. Bardzell, drawing on feminist philosopher of science Sandra Harding, argues that engaging in politics can offer "new avenues of insight" into the organization of social life [8]. Ethics too can be part of politics; political ideals often require forms of ethical judgment and action by those who belong to the collective [54]. In HCI, scholars often investigate ethics as part of the research process or the harms of the final design, while others might count systems design processes themselves as part of the practice of politics that contain implicit ethical norms [83]. This paper focuses not on the politics of design, but rather, the ethics and politics of qualitative research realized to inform the design and operation of a highly-complex, real world socio-technical system - Wikipedia.

\section{Care}

Our approach to ethics and politics draws heavily on the feminist ethics of care [91, 118, 137]. Care orients researchers to embodied sensitivity, experience, affect, and the provisioning of resources and knowledge [38, 68]. Care has a long history in feminist studies of science and technology as a model form of engagement that privileges the work of nurturing, cultivating, sustaining, and maintaining [37, 103]. In this way, science and technology studies scholars pose care as a corrective to cultural emphases on discovery and invention - origin moments that neglect the ongoing labors and attention to the mundane that keep our material, technological, and social world going $[46,84]$. In the face of 'ethicopolitical' questions, care ethics do more than show concern for a techno-scientific controversy [96]. They engage in public action around these concerns [44], but go beyond to take responsibility for locating those things 'neglected' in social life [37].

Care ethicists argue that researchers must recognize that their "ways of studying and representing things can have world-making effects" [95]. Research does more than represent the world out there [135]. Researchers represent, make new social relationships, participate in issue and public formation, and can obscure or exclude as they assemble. As a feminist commitment, care calls on researchers to go beyond questions of truth and bias, to ask how research can generate 
care and help others care $[40,101]$. Yet care is not innocent. Attempts to care can also play out as domination of a carer's vision or agenda over those of the cared for [112]. There are many ways that investigators activities and investments might manifest care. We draw from feminist ethnography to understand the forms of care research participants might enact as active agents and knowledge producers.

\section{RELATED WORK: REFLEXIVITY AND COLLABORATION IN QUALITATIVE RESEARCH}

In socio-technical research, there is a long tradition of ethnographic methods [116, 134]. In recent qualitative, socialcomputing work, digital ethnographers have engaged with traditions of feminist ethnography, and have intervened in the lack of recognition for the ways identity, culture, and power are configured in socio-technical systems [9, 60, 122, 145]. These interventions have taken a number of forms, including the outlining of reflexivity: the situated practices by which researchers attend to their own embeddedness in the practices, institutions, and power relations under study. Reflexive researchers intentionally cultivate sensitivities to how this embeddedness affects their relationships with subjects and the knowledge they generate through their research $[108,128]$. Conversations about reflexivity differ from those of bias. The language of bias builds on the values of 'fairness' and 'objectivity', but conversations about reflexivity focus on how knowledge-creation is always a site of culture, power, and labor.

From HCI and CSCW, work in participatory design in action-oriented research in grassroots, and organizational contexts $[15,16,48,126]$ social justice, and community-based design $[45,55]$, has also illustrated the research process as an ongoing activity of learning and cooperation. Projects in these areas have shown how treating participants as active shapers of research design can alter and enhance how tools and systems are made, and how they enter the world.

In the field of Anthropology, discussions of reflexivity and positionality grew in the 1970 s, during what's called the 'reflexive turn' or 'postmodern turn.' Clarke and others identify this moment in anthropology as a response to decontextualized, structural, and humanistic narratives in canonical early works in the social sciences and cultural anthropology, which focused on cultural difference and were tied to colonialist projects [30], such as in the fieldwork of Franz Boas, Clifford Geertz, and Claude Lévi-Strauss. Scholars in this turn recognized the politics of how ethnographers represent the Other, in everyday life or across scales of culture or nation, as well as the fiction of distance in many cases between researcher and researched [1]. Feminist analyses influenced this turn by showing how the positionality of the researcher, in relation to subjects, is conditioned by gender, race, class, disability, and local and transnational histories
$[70,72,117,140]$. Some feminist anthropologists have questioned whether the colonialist legacy of ethnography cannot be separated from the ways that the ethnographic method focuses on representing difference [73, 131, 141]. The feminist sociology of knowledge suggests that the history of the science and the epistemologies of objectivity that have also appeared in the social sciences, are steeped in their relation to power - where claims to objectivity instantiate existing social orders of governance and rule [130]. Today, anthropolgists understand fieldwork as the production of knowledge-generating encounters between differently positioned participants and fieldworkers whose relationships are shaped by language, history, and power.

The rise of the internet and economic globalization challenged anthropologists to rethink the pacing and spatial organization of fieldwork. Colonial ethnography assumed fieldworkers came from worlds disconnected and more modern than those people they studied. Globalization and critiques of colonialism instead showed the longstanding connections between 'modern' and 'traditional' worlds. Holmes and Marcus call for ethnography that can engage experts not as subjects of worlds disconnected from fieldworkers but as participants with whom to stage an ensemble of explanatory connections $[78,79]$. Coleman, studying Anonymous, described the challenges of representing a group whose cultural practices hinge on performing as an anonymized multitude [31]. Lincoln and Denzin encourage anthropologists to describe and critically analyze their own engagements in policy, mentoring, activism, and community involvement around their topics of interest and to subject these para-ethnographic and collaborative engagements to critical rigors [39]. Nardi et al. offer distinctions between "participants," "key informants," and "collaborators" who actively shape research [18]. These perspectives demonstrate the potential for qualitative research to reimagine the roles, responsibilities, and organization of research in socio-technical worlds.

\section{HOW CARING ABOUT WIKIPEDIA BECAME CARING ABOUT GENDER}

The insights of this paper have developed from the first author's involvement as a Wikipedia editor and research subject in multiple studies on Wikipedia. In this paper, we reflect on the social and methodological implications of what was observed during this research participation.

Wikipedia has long piqued the interest of researchers, who have used it as a petri dish to study emergent modes of collaboration, creativity, and computer-mediated communication. But social scientists and HCI scholars have expressed different reasons why Wikipedia is important, and what it can tell us about socio-technical systems. Overall, Wikipedia occupies a different theoretical space, and is used to ask different questions, within the social sciences and within HCI. 
In the social sciences, Wikipedia has been studied within the ecology of Free/Libre software and Open Source Software (FLOSS) projects like the GNU/Linux operating system, Usenet, or the Apache web server, as well as open access projects building capacities to resist the restrictive nature of intellectual property law [89, 120]. Some social scientists have investigated Wikipedia for its unique political culture as a social movement and site of activism and collective action, as well as its practitioners' resonances with the 'hacker ethic' $[31,75]$. Others have explored the power dynamics reflected in the social formations between editors and infrastructures, including Wikipedia's meritocratic culture, reliance on the free labor and resources of volunteers, and parasitic relations with search engines and the corporate tech industry $[86,138]$. Yet others have used political economy, law, economics, and communication to describe Wikipedia as a cultural and information 'commons' and applied economic and behavioral principles like altruism, good faith, reciprocity, and gift-exchange to understand it in broader cultural contexts [10, 26, 29, 76].

On the other hand, while a large and growing body of work in $\mathrm{CHI}$ focuses on Wikipedia, $\mathrm{CHI}$ papers contain very different justifications of why Wikipedia matters. One prominent way CHI researchers incorporate Wikipedia into their studies is purely technical: it is a reliable source of large-scale open data sets to use for any number of technical problems, as well as to study social aspects of online interaction [125, 142, 147]. Wikipedia has been especially useful to computational social scientists using big data to study and visualize the predictive relations of interactions on Wikipedia such as edit histories with geopolitics, natural disasters, and elections [6, 64]. Others in HCI have both quantitatively and ethnographically queried Wikipedia as a test bed for theories about platform governance, crowdsourcing, social networks, collaborative computing, cooperation, group work, moderation, platform retention, and information credibility judgments $[5,11,66,93,94,105,139]$. It has also been contextualized in terms of crowdsourcing and to explore topics related to crowdwork, that come together in microwork platforms like Amazon Mechanical Turk [92].

Wikipedia is sometimes thematically grouped with usergenerated content platforms like Youtube and social media sites like Facebook in studies of online communities [87]. Characterizations of Wikipedia as a social media platform are harmonious with danah boyd and Nicole Ellison's often consulted definition of social media: online services in which people can construct user profiles associated with an identity, connect and form relationships with other users, and message, and comment [20].

The questions of social science researchers and HCI researchers meet in the study of conflict and deliberation on Wikipedia. Although one of Wikipedia's mantras is that 'anyone can edit,' much research has found that Wikipedia's editor community lacks demographic representativeness. A 2010 study was the first to report that there was a disproportionate amount of women Wikipedia editors - its results estimated that this number hovered around 13\% in the English Wikipedia [63]. The 'gender gap' research that emerged after this 2010 study most often approached systemic bias and diversity in two ways, sometimes proposing forms of causality between them $[47,77,94]$. The first was to highlight how the gender gap emerged from Wikipedia's adversarial community culture, and could negatively impact existing women editors [32, 53, 93]. A second approach hypothesized that the gender gap produced insufficient coverage of women's issues on the encyclopedia [119].

Some research on gendered dynamics in Wikipedia and in the open source community has focused on gendered socialization and access to technology in STEM fields [74]. However, gendered interaction also takes on a unique valence on Wikipedia based on the affordances of the platform. Some reports indicate that women Wikipedia editors are deterred by increased incidents of harassment, and have investigated if and how harassment may have been enabled by the socio-technical affordances of the platform.

These studies have often defined conflict according to discreet forms of interaction and social order between users and the platform, and then analyzed the dynamics and occurrences of those modes. [61, 139, 148] For example, Wikipedia 'edit wars' - high-frequency conflicts in which editors aggressively revert or add content, are often visited as a cornerstone of Wikipedia conflict $[67,148]$. Other research has pointed to Wikipedia's culture of treating new editors as second-class citizens before they learn to apply Wikipedia's many complicated rules. Community members have often concluded that editing Wikipedia requires 'thick skin' to deal with the confrontational and aggressive interactions it can invite.

In 2014, a series of large-scale cross-platform coordinated attacks against women, including 'Gamergate,' changed the context and urgency of work on online controversies and harassment. These attacks were led by men reacting to changes in gender dynamics in the video game industry and criticism from feminists and others about the embedded misogyny in gaming culture. [102] An outspoken Gamergate target feminist video-game blogger Anita Sarkeesian described some of the attacks done to Wikipedia articles on women, including herself, which included vandalism such as additions of gendered and racial slurs and pornographic content [124]. These events sparked a wave of research on online harassment across social-computing fields, as well as a rise in organized efforts, activism, and public discussion of online harassment $[47,107]$. It was around this time that the Wikimedia Foundation began to produce reports on gender and Wikipedia, hired staff to focus on community 'safety and support' namely, to 
mitigate harassment on Wikipedia and at community events, and built grant programs to fund projects that proposed building tools to address Wikipedia's gendered dynamics $[2,115]$.

These different landscapes of research on Wikipedia in the social sciences and in HCI have grown substantially in the past decade, and we can imagine that they will continue to do so given Wikipedia's sustained role in international, multilingual, information ecologies, including the dependence of companies like Facebook and Google on its results.

\section{BECOMING THE SUBJECT OF RESEARCH}

It was 2013, and I was working at a non-profit organization in New York as a Wikipedian-in-Residence, a position which involved liaising between Wikipedia community organizations and libraries, archives, and museums. I spent my time divided between encouraging the adoption of open licenses for institutionally-owned materials like photographs and metadata, and organizing 'edit-a-thons' - participatory, instructional technology workshops designed to encourage people to become Wikipedia editors. I was also speaking in public forums and blogging about issues pertaining to Wikipedia.

One day, I found a message in my email inbox from a researcher who had found my name on a Wikipedia-related mailing list that I posted on from time-to-time. The researchers stated that they were working on a project related to experiences of gender and harassment, and that they wanted to interview me. I responded enthusiastically: at the time, I would talk Wikipedia to anyone who asked because I felt it was my mission to spread the word. I had also never been in a study before and was curious about what it entailed. The following week, I had a Skype conversation with a graduate student researcher who wanted to interview me for their study on editing behaviors and conflict on Wikipedia.

This was the first of many ethnographic studies about the dynamics of Wikipedia editing that I was interviewed for, of around 10 in total over the course of three years from 2013-2016. Between responding to emails, signing consent forms, setting up and having the interviews, and responding to follow-ups, I spent hours doing the work of a research subject. Despite the commitment of time and emotional energy, I did all this because I felt that volunteering for the study opened a wider of a conversation that I could take part in. The study, in turn, could contextualize further debates about diversity in the community. The reflections that follow have been reconstructed through memory and corroborated by email archives. I did not take field notes as I had these experiences. What follows is the outcome of several years of feminist engagement and reflection on my experiences, leading me to denormalize my research encounters and reflect on how they could have proceeded otherwise.

To be a woman editing Wikipedia was one thing. To be a woman participating in research was entirely another. As a research informant, my day to day practical experiences of Wikipedia work became resources for researchers - budding experts who promised to generate general knowledge or at least middle-range theory [27] to explain the social processes that produce Wikipedia's gender and broader diversity dynamics. In what follows, we retell several moments that show how research subjects do not only tell their experiences, but manage and navigate the research process.

\section{WHEN RESEARCH SUBJECTS CARE}

Since I began contributing to Wikipedia around 2009, gender noticeably shaped of my interactions on the platform. My experiences included many hostile dynamics that others have reported and observed, including extra scrutiny of my contributions. These negative encounters with other Wikipedia volunteers challenged my ability to edit and correspond as I wished in community channels like mailing lists and IRC bulletin boards.

These issues were of concern at both personal and professional levels. I felt deterred from contributing to certain areas after being harmed in those spaces. As a result, I worried about recruiting women to participate, when they would quite possibly experience gendered challenges in a community that lacked the organized infrastructure to address these issues. Hence, the longer I spent editing Wikipedia, the more motivated I was to do something about these issues. I channeled this drive into community organizing work and enthusiasm for research study participation to contextualize community discussions of gender and diversity.

In 2013, as a Wikipedian-in-Residence and community organizer affiliated with Wikimedia New York City, I began initiating edit-a-thons that focused on writing Wikipedia articles about women. Wikimedia NYC collaborated with libraries and archives to produce lists of articles to add and resources we could cite, and worked with people to develop positive experiences editing Wikipedia. These events multiplied, and gender and Wikipedia became a central component of my work. From 2013-2015, I worked with others to organize many such events, including a series of international edit-a-thons called 'Wiki Loves Pride,' and 'Art+Feminism,' presenting at Wikimania - Wikipedia's international, annual conference, and at other events.

This personal experience and organizing work around the gendered aspects of Wikipedia fueled my interest in participating in research when I was contacted for interviews on this topic. At the time, I was already reading the research on Wikipedia to help inform my organizing work so I would be able to explain the issues to people that were interested in getting involved. As organizing groups and committees, we discussed feminist work about online culture and gendered harassment. We sought, together, to support our understandings of the potential impacts 
the events we organized, including impacts on those of us who were volunteering our own collective energy and labor.

Concurrent with this event organizing, researchers recruited me to participate in research studies - most of them related to gender and Wikipedia. I personally believed that more research on the topic would further prove the urgency of the issues we were organizing to address. I was eager to participate in these studies and found them useful to understand how these researchers, with varied affiliations and projects, framed, described, and analyzed the issues. I saw being a research subject as an extension of my activism that might eventually circle back around and contribute to the organizing work I was already doing in the open source movement. My confidence that participating as a research subject might affect issues I cared about was further reinforced in that I already recognized some of the researchers who contacted me. I inferred their agendas and potential impact by seeing what conferences they participated in, who they were cited by, and even how they contributed to projects directly on Wikipedia - writing content, adding images, and so on. I believed that the published reports of these studies would form a basis for future grants and community efforts. Their research would legitimate the efforts I wanted to see happen.

Over the course of participating in multiple studies, I felt I was learning about how to be a research subject. I developed an acute, understanding of how to navigate the interviews. By doing one, I was also in a sense, practicing for the next. For example, when asked the generic, open-ended ethnographic interview questions like, "What has been your experience with gender and Wikipedia," or "What do you find difficult about participating in Wikipedia," I spoke strategically. That is, I relied on my expertise and even mentally prepared for the interviews beforehand, because I wanted to lay the groundwork for changes I wanted to see - changes the research could legitimate. I made a checklist of things I wanted to get to during the conversation; I prioritized.

In the interviews, I spoke on a range of topics, including how I was deterred from editing certain topics for fear of the negative attention such edits would draw to myself. Another theme I could speak to was Wikipedia's meritocratic culture. In this regime, I detailed how women (myself included) are accused of not having high enough 'edit counts' to engage in certain conversations, be elected as administrators, or to speak authoritatively about how to apply Wikipedia's rules. These exclusions mattered for representation in the encyclopedia as well. Women were often excluded from 'articles for deletion' (AfDs) debates organized to resolve conflicts over relevance and notability. Moreover, I detailed my own experience in which my negative social experiences on the platform led me to focus on less scrutinized work, such as creating 'stubs' - small articles on innocuous topics - or doing community organizing work.
I avoided working on widely-read, but very hotly contested articles, like those on 'feminism' or 'Kim Kardashian.'

The first author's experiences represent some of many potential reasons why people might participate in research studies. The observations above show how a subject's willingness to participate in a study might express wider social projects and even serve as an activist method to create platforms for discussion. People come to find meaning in research by engaging with it to benefit their communities even if they don't necessarily directly benefit themselves. Research subjects can discursively scrutinize research settings as political sites to articulate their perspectives in the world. By understanding the research setting in this way, we can inquire into how subjects understand, as insiders, how their storytelling might affect the social issues and communities they care about.

Research subjects are not empty canvases. As a participant in multiple studies on Wikipedia, my understanding of the research encounter as a site evolved over time. However, the researchers who asked me for interviews treated me as a repository of knowledge rather than as an active agent that might selectively engage in the act of telling [4]. In treating me as such a repository, they had to make several assumptions. First, they treated my labor of narration as free. Second, they interpreted my stories as evidence that revealed the symbolic structure of my social world [17]. Through conversation, they seemed to assume, I would reveal the workings of a place they did not intimately know, including what I found most useful for them to write and spread the word about.

As a research subject, I was also able to detach myself from my own subject position to think about the context of the study from the point of view of the researchers. This thinking was part of my cognitive, psychological experience of being in a structured relationship in which my reality would be scrutinized and subsequently turned into social science knowledge and expertise.

Researchers might have engaged me differently if they understood me not only as a repository of experience, but an actor in the organization working to change the very dynamics they sought to characterize. If researchers were to take this into consideration, perhaps they might have invited me to suggest areas of inquiry for them to attend to. What would have been different if they had asked what concepts or processes I wanted emphasized in research production? Researchers, with their powers to anonymize and take the heat for research findings, can often make contacts and circulate stories people otherwise would not share widely. This unique positionality has great potential if researchers consider how they might not only care for subjects, but care with them. 
My experience though unique, is a case of a wider phenomenon of research subjects who participate in reflexive cultures - cultures in which participants seek to reflect on and reform the culture itself. HCI researchers might ask how to care with subjects in the ways we suggest when they study those who see themselves as responsible members of a community of production, for example Wikipedia participants, hackerspace members, or patient activists.

\section{CLOSE CONNECTIONS}

Close ties in the social worlds researchers seek to understand can also complicate matters for research subjects. These ties might be constituted by prior relationships or the building of ties during the research process.

Although I was almost always more than happy to talk to researchers, there were times when I was also concerned about the overlapping social networks and personal ties that I had or might have with them. Wikipedia researchers are often active participants or editors of Wikipedia. When signing up for studies, I often knew that the researchers I had talked to were only a stone's throw away from peers and collaborators. In fact, the researchers that interviewed me were sometimes people that I knew or knew of before agreeing to participate. They were friends of friends perhaps, people that had been given my name or contact information by someone who thought I was a good candidate for the study.

When I was volunteering for these studies, I was concurrently talking to other Wikipedians whom I was close with about our experiences being research participants. Apparently, I was not the only one who kept being called upon and agreeing to be a research subject. In fact, I was one of many - there were a circle of people who were active across projects relating to gender and Wikipedia and frequent collaborators in event organizing and online initiatives. Many of them had participated in the studies I had. The same circle of organizers were also the frequent posters on mailing lists and public channels where people talked about gender and Wikipedia. These channels were composed of self-selected people interested in topics researchers were looking into. So, perhaps it's not surprising that the participants in these mailing lists often were recruited to be research subjects.

One site in which research subjects reflect on their own experiences is by following up with research studies they have been in and drawing connections between research and their own lives and communities. For example, I have read all the publications I've been characterized in. Because of my privilege as a student with institutional access to journals behind the 'paywall'- a scholar in training - it hasn't been difficult to locate the publications myself.

My interest in reading the final products of the studies I have participated in has been motivated by several factors, including my general interest in the topics. Another factor was my concern about how I was portrayed. Reading something about yourself or something in which you are quoted in can be a scary experience. Sometimes the results are satisfying, other times disappointing. I felt that many did not portray what I knew in ways resonant with my self-identity, nor my understanding of my community. I felt frustrated when the concepts that I thought I clearly had explained were given other names or grouped into categories that I didn't identify with.

My hesitations about the validity of the findings commingled with disappointment that the investigators had not involved me in the research process after the interviews. On the contrary, it felt like a surprise to read the publications months after the interviews were conducted. Although some researchers said that they would inform me when the publication had come out, no one ever followed up with this promise. I counted this as a missed opportunity for them to deepen the usefulness of their findings to the Wikipedia community.

There are many attitudes which CHI researchers might bring to the table when they attempt to manage the effects of their work on research subjects, scholarly communities, and institutions. In ethnographic traditions in the social sciences, transparency and the development of trust are enmeshed in debates about the constructions of best practices. These might include the production of archives - perhaps open or perhaps for the community - so materials gathered in research can be used by communities involved. These practices might also include collaborating with communities to produce ethnographic documentation $[69,99]$.

One of many such routes is called member checking - a term for a cluster of procedures that researchers can employ to consult with participants and clarify what they have conveyed in interviews and other documented interactions [100, 127]. Member checking techniques might involve the recording and transcription of interviews such that researchers can play back recordings or share transcripts with participants to allow them to retract or clarify aspects of what they originally stated on the record. The concept of member checking is a framework for managing the inherent power differentials between investigators and participants by generating opportunities for dialogue about the (mis)interpretation of findings [88]. Member checking might further call on researchers to share results with communities who helped them produce the findings, such as through distributing a finalized publication in a translated and accessible format.

Member checking might have mitigated the frustrations I and others felt by including us in a dialogue about how our worlds were interpreted and represented in research. This process might have also helped participants understand how the aims of the research evolved over the course of the research as researchers learned more through their inquiry.

What other forms of research-subject interactions might have been more encouraging and validating? One next step to complete the loop would be for Wikipedia researchers to 
interact with subjects in ways that convey that they don't just want to extract information from participants so to analyze them as apolitical objects, but to impact the issues that they are generating knowledge about [109]. This approach would recognize participants as active agents who also wield different powers of representation and accountabilities. This step is especially crucial for researchers intervening in topics where participants already represent their own and group interests, such as inequality and diversity on digital platforms. Researchers must account for how their own knowledge production and circulation participates in a wider public of which their research subjects are also a part. Researchers producing reflexive knowledge for powerful institutions such as Wikipedia tread a more complex path in which their research wields greater impact than much qualitative academic work. And in communities with close connections, these research interventions not only represent the world but very immediately transform delicate social relationships through which the community operates.

In communities with close connections, recruitment processes can also produce anxiety or even risk. When researchers contacted the first author during recruitment, they asked her if there were other people that might fit the parameters of the study. This recruitment tactic is known as 'snowballing,' - the practice of recruiting participants by having others share information about others who may contain the 'trait' of interest to the study, and is extensively used in CHI [23]. Some snowball sampling methodologies include ethical contact and transparency guidelines for communicating with research participants $[12,21]$. Still, snowballing asks a troublesome favor - the disclosure of identifying information about members of their social networks who may or may not wish to be contacted and roped into a research context. The first author was torn between wanting to help researchers and being concerned about how her relationships with others might be affected were they to be told that someone had given the researchers their contact information because of assumed aspects of their identity or experience. Snowballing asks participants to reveal identities of people who might not want to be found or are not interested in engaging. These risks run high when the topic touches on sensitive and private issues - for instance recruiting participants for a study on online harassment.

A more participatory approach to producing HCI knowledge might mitigate these risks by involving subjects in crafting the goals of the project and the senses of safety that shape research protocols. We draw inspiration from participatory design - a tradition originating in the 1970s in Scandinavia that has inspired practices of making, of theorizing, and research methods, in many areas of design, including HCI $[44,111]$. Participatory design foregrounds researchers' effects on the communities they engage with, and the critical, political potentials of design to impact society, particularly in everyday interactions, by enabling social change, class solidarity, and participatory democracy. Muller and Druin have described participatory design interventions in HCI as an in-between or 'third space,' that gets at the complex encounters and experiences of intimacy and vulnerability between researchers and participants in the research setting that can be built into the design process through community imagining, collaborative ideation, and co-creation [108]. Participatory design has expanded the locus of its work from workplaces to communities [36], producing a rich literature on how designers can facilitate community interactions with organizations, institutions, and government.

A participatory design orientation to gender in Wikipedia opens up questions about how the institution works, how people have been trying to influence it and failing, why and how people are excluded from decision making, and how participatory design practices can produce new forms of collaboration, power building, and influence. Gender researchers entering the fray of diversity work in Wikipedia might go beyond the production of new facts, critique, or expertise [96] to instead generate stronger publics around issues of gender. By publics, we mean groups of people articulating common concerns [36] and mobilizing within or even beyond formal or legitimate institutional channels [7].

Although there are hundreds of thousands of Wikipedia editors on the English Wikipedia alone, the number of people who were organizing 'edit-a-thons' with cultural institutions, and engaging with conversations about gender and Wikipedia was smaller. Many already had close connections. Researchers can broker sensitive knowledge among these communities by creating participatory but safe spaces, by documenting and aggregating knowledge in collaboration with these highly invested publics, and by offering analytic insights that might help these publics achieve their political goals. Where qualitative research often produces what Lucy Suchman has called 'detached intimacy', participatory processes have the potential to draw the researcher into "located accountabilities" of technological - and here, organizational - production [133]. These located accountabilities recognize that knowledge is partial, situated, and personal if structured by power; researchers and participants alike take responsibility for their knowledge and act collectively to produce a new kind of situated knowledge that can change the community's conditions [70].

\section{THE LABOR AND PAIN OF SPEAKING FOR OTHERS}

A fundamental aspect of qualitative research is that investigators develop theories by abstracting from information gathered from a limited pool of subjects. For research participants, speaking for others can be especially stressful when it isn't 
just a political abstraction, but involves actually speaking for other people we know. But participants are knowing subjects - aware that the knowledge they convey and produce in their interactions with investigators will be generalized, and that this will affect their communities.

Researchers often assume that participation is not work, and that people contribute their time to research to contribute to broader knowledge. IRBs may actually forbid paying study subjects for their time as workers for fear of "undue inducement" or coercion - motivating people without intrinsic desire to participate in knowledge production. However, when structural and political conditions make certain people marginal, it is often those people who bear the burden of participating in research, remedies, and scrutiny in public debate.

At various times during research interviews, I became concerned that, by being a research subject, I inadvertently might be speaking on behalf of many. Situationally, this discomfort unfolded in two different ways. The first was when I was asked questions that were about my own experiences. My Wikipedia organizing created contexts in which my stories were already made public because of my public role in speaking about gender and Wikipedia at events. I believe that my research participation further amplified my voice as a source of expertise. The second source of pain was that I knew that this phenomenon was not unique to my own experience.

During the interviews, when I was asked questions about myself, I was worried that they would be generalized to apply to 'women in Wikipedia' as a broad social group. In one study, I was asked, "why do you think some people are targeted with online harassment?" - I believed that they were saying 'you' but were going to generalize to "this is what women say about online harassment" in their report. That's what their study was about, according to the information sheet they had given me as part of the consent process. My apprehension and anxiety about balancing my self-representation and making generalizations about my community was further consolidated because of the subject-matter I was being asked to talk about. Making generalizations about experiences with gendered harassment is a fraught and tenuous request. If that was to be my task, how was I to know how my ideas might be abstracted and operationalized?

I was also asked questions in research interviews about what I felt were common trends across the Wikipedia community. In some ways, I had talked to many people about their experiences and perhaps contained some expertise to be able to make informed generalizations. On the other hand, I felt concerned with the implications of my voice being heard louder than others because of the influence of research on other infrastructures, including further research attention and funding. I felt I needed to balance telling my own story with explaining how that fit into broader trends and conversations I noticed based on my social position and networks. I was also unsure how to situationally convey that I could not speak for others. I felt a sense of duty to tell my stories in a way that conveyed what I didn't know. Further, I was worried about managing how the stories I told researchers would affect how Wikipedians were going to be portrayed in the research and further how this research might impact the Wikipedia community.

I had multiple experiences with gendered harassment on Wikipedia. These events occurred both at in-person events and online and took myriad forms, including online trolling, mocking for lack of certain technical skills or Wikipedia policy knowledge, and unwanted advances at in-person events. And yet, because Wikipedia is by-and-large managed by volunteers, many people in the Wikipedia community, including myself, had already made complaints about the lack of infrastructure or standard procedures for dealing with harassment complaints in the community.

It is not always easy to predict how you will respond to questions regarding experiences that were traumatic, and responses to trauma do not get more predictable over time. Doing interviews about gendered harassment was hard. I sometimes surprised myself, expressing intimate emotions or breaking into as tears, all on the record. The embarrassment I felt about such expressions of emotion bumped up against the cathartic. I also found the comportment of the researchers - who were in a procedural position that theoretically constrained them to remain impartial listeners - to in fact be sympathetic.

But despite the emotional difficulty of recounting these experiences, I enjoyed being a research participant. Complaining about a negative experience got it out in the open. Telling my stories in a private context in which my experiences could be validated was relieving.

Described above are multiple discursive processes in which subjects might come to understand research settings at intimate, psychological, and inter-subjective registers in the process of telling, recounting, and translating prior experiences. Qualitative interviews create contexts for active listening that might impact participants emotional experiences and interiority in any number of ways. These contexts are complicated: they involve answering personal questions that are designed to elicit responses that can be abstracted into generalizations. However, existing assessments of the emotional work of research tend to focus on the wellbeing of researchers in 'sensitive settings' rather than participants, including how researchers can engage in 'boundary-setting' [41, 42, 104].

Research participation can require subjects to relive intimate experiences. But when the topics touch on things that were difficult for us to experience, qualitative interviews can also trigger negative affect or even trauma. However, as our examples indicate, sometimes people engage in research studies that they believe might affect the issues they care 
about, even when telling stories about experiences that are personally painful contains complex risks.

Being a participant in qualitative research often involves being asked to talk about one's personal experience, so that researchers can compare multiple accounts to make generalizations about groups [4]. Power, politics, and capital affect which voices are heard and amplified to speak authoritatively, and whose representations of the world researchers count as legitimate information [132, 146]. Much critical work in critical race studies, feminist studies, and education have depicted ways that people do work that sometimes involves personal or political risks to engage in the politics of representing themselves and teaching others to recognize differences that they have been personally affected by $[52,57,80]$. Many have also described how people who have experienced oppression can grasp complex structural issues and relations someone who didn't live those traumas might not be able to access. Sara Ahmed asks, "who does the diversity work?" and considers "how diversity work becomes associated with certain bodies," by tracing a genealogy of the incorporation of diversity rhetoric and recent, mainstream institutional interest in diversity and inclusion [3]. In other words, the responsibility of speaking out against issues of social and structural racism often falls on the shoulders of those who have already experienced those same forces of marginalization.

Marginalized populations face increased risks in participating in research that might make aspects of their identities public. For example, some Wikipedia editors are especially worried about anonymity - for example, LGBTQ+ editors in countries where being out is more of a risk of being susceptible to violence. This example demonstrates a scenario in which subjects can be self-selecting in that volunteering for studies might involve us 'outing' ourselves to researchers, and to others. Even when researchers are obligated to procedural ethics required by research ethics review boards like the IRB, participants' concerns do not necessarily disappear with informed consent procedures and institutionallymandated privacy assurances [110].

To what degree might researchers be aware of the potential impacts of research participation on subjects? What should HCI researchers do about the unpredictable but potentially meaningful emotional experiences they may elicit during their work? What are alternatives to asking people to talk about potentially traumatic experiences? Ellis discusses the intimacy built between research actors, and suggests that a relational, rather than procedural ethics can better encompass the affective responsibilities of researchers to manage the human relationships that form during research. Etherington suggests informed consent as a relational process rather than a procedural event [51]. Many have also described how research can produce therapeutic encounters, and engaged with autoethnographic writing in which researchers describe forming friendships with participants $[33,49]$. Birch describes how qualitative interviews that involve 'acts of self-disclosure' involve managing intimacy, rapport, and professional boundaries at once [13]. We suggest that HCI researchers maintain awareness about the complex ways that a range of emotions, including harmful ones such as traumatization and re-traumatization might look or happen to both subjects and researchers, and how this harm affects inclusion, to develop access to support, guidance, and protection within our scholarly communities. Researchers can learn from considering the emotional stakes of research participants to understand the ways that ethics are co-negotiated entities rather than immutable approaches.

\section{CONCLUSION: NEGOTIATING ETHICS}

The stories we have told detail how research subjects have complex relations to the processes of producing scholarly knowledge. These forms of knowledge production grow in the contexts that research creates. Subjects develop complex, psychological, interpersonal, and political relationships to research infrastructures, as they make sense of the instruments that participating in research affords [97]. Thus, research settings are sites of the negotiation and interpretation of meaning, that create opportunities for rich discussion and involvement of subjects in the future possibilities for the research and how it might affect the world. Researchers and communities that help produce research live in this world together.

This requires a notion of ethics not as a set of pre-given principles, but as a negotiation in which subjects and researchers work out an ethical, accountable practice or project together. Here, we draw on feminist STS approaches to ethics as a system of agential relationships that cannot be assigned to 'unitary subjects' such as administrative checkpoints like the IRB, codes of conduct, or individual choices [71, 109]. As $\mathrm{HCI}$ researchers converge to refine the IRB process to better manage ethics and accountability in research on emerging technologies [110, 136], feminist STS can also support wider conversations about ethics as constantly negotiated agreements distributed among communities of practice who create shared understandings in specific, power-laden contexts. But these responsibilities are also not always equally distributed - some take on more labor than others to manage ethics or bear harmful consequences [3]. Critical geographer Sarah Whatmore describes this phenomenon as the distribution of ethics across scales of space and place - where the psychological, social, and political are also networked and transformed by geographical configurations [144]. For instance, ethics - and who bears ethical responsibilities - is culturally and geographically situated, and these ethical meanings are not 
universals, but situated in place. Different cultural formations might formulate ethics as democratic rights in liberal projects, freedoms of identity and expression as in some feminist thought, or be extended to the non-human in ecological and environmental philosophy as in Latin American decolonial thought. Working from feminist epistemologies, we can get at more engaged forms of ethics by understanding the emplaced accountabilities in the working relations between researchers and subjects [133].

Ethics, then, is not universal, but situated. We suggest care as a way of navigating this negotiated, contested ethical field. On the one hand, care is a culturally, affectively and socially-situated practice of knowing and interacting in relations by maintaining sensitivity and attention, rather than a formation of ideals. By contrast, values, morals, and ethics are systems of principles that define and are fastened to achieve 'the good' - an abstract concept for a just world. Although they are sometimes used interchangeably, we suggest that care represents a different, but interlocking analytic system than those of ethics, values, or morals. Further, we argue that it is useful for HCI practitioners to examine the tensions between ethics and care because these ways of interacting with the world move through different political constructions of the meaning of 'social good,' as they are enacted - by individual decision-makers, groups, or institutions. In this context, care can be a practice of recognizing the reality of social facts and intervention in lived, realized, social, political, and scientific realities and doing something about it [38].

Future work might explore cross-overs between our discussion of care and research participation and bioethics literature on why people have voluntarily participated in research, including as a form of the expression of self-determination, moral responsibility, and even grassroots activism [106]. For example, Morris and Bàlmer describe how people negotiate their roles in research in real time as they manage the research encounter, accomplishing roles as varied as 'giver', 'patient-beneficiary', 'client', 'collaborator' and 'guinea-pig' [106]. Gere and Epstein discuss grassroots organizing and the movement for patient autonomy in medical testing, in which AIDS activists claimed a right to participate in risky, experimental HIV-AIDS research [50, 62]. Cox and McDonald conducted interviews with people who had previously participated in multiple forms of health research, and a number of ways participants might understand their own responsibilities as a form of active, ethical engagement, including 'volunteering' and 'being a good citizen' [34]. As computing technologies and medical technologies converge in some cultural understandings of human flourishing, well being, addiction, or trauma, HCI debates on ethics might fruitfully learn from longstanding conversations on socially constructed and contested biomedical research encounters.
Researchers do not only represent. They also intervene in and disrupt sites of study - what Müller and Kenney call 'interference effects' [109]. Research products engender multiple publics that come together to produce knowledge through the collective labor of subjects, investigators, and others who labor to support them. Such moments are opportunities to reflect on the ethical possibilities of conducting qualitative research that acknowledges the ways that research insights are already collaborative and negotiated efforts in which participants may seek agency over the potentials of research outputs.

These interference effects are features of all knowledge production, but we believe these effects are particularly felt by participants of reflexive cultures - cultures in which participants seek to reflect on and reform the culture itself. When HCI researchers engage people who see themselves as responsible members of a community of production, for example Wikipedia participants, hackerspace members, or patient activists, they ought to expect that their research not only represents or intervenes but also creates new circuits in reflexive cultural contests. Culture is not simply "out there" [135], but made, in part, through here as we inquire, write, and publish.

The intervention of this paper is to consider how the exercise of politics by research subjects might lead us to understand how subjects and researchers can work together, and how this can help researchers more deeply understand their experiences of producing data. We have suggested that research subjects have intentions when they enter relationships with researchers that can include politics, representation, and trauma. This paper raises difficult questions about the interpersonal, institutional, and discursive landscapes that research subjects traverse. Exercising care towards research subjects can impact the use, application, and sustainability of research, research products, and their pedagogic afterlives. As HCI attempts to care for people amidst technological transformation, we must match that care with care for the lived experiences of people we engage through our methods along the way.

\section{ACKNOWLEDGMENTS}

The authors would like to thank members of UC San Diego's Feminist Labor Lab and Design Lab for workshopping these and other ideas. We are particularly grateful to Davide Carpano, Sarah Fox, Akshita Sivakumar, and Veronica Uribe-del-Aguila for reviewing drafts. Thank you Os Keyes and Sucheta Ghosal, whose work and conversations inspired some of the ideas in this paper, and to Baran Kiran. We also thank Camille Nebeker and anonymous reviewers for careful readings and comments that greatly improved this work.

In loving memory of Dr. Matthew O. Howard. 


\section{REFERENCES}

[1] Lila Abu-Lughod. 2008. Writing against culture. In The Cultural Geography Reader. Routledge, 62-71.

[2] Wikimedia Foundation Community Advocacy. 2015. Harassment survey. Retrieved December 14, 2018 from https://meta.wikimedia. org/wiki/Research:Harassment_survey_2015

[3] Sara Ahmed. 2012. On Being Included: Racism and Diversity in Institutional Life. Duke University Press.

[4] Linda Alcoff. 1991. The problem of speaking for others. Cultural Critique 20 (1991), 5-32.

[5] Judd Antin. 2011. My kind of people?: Perceptions about Wikipedia contributors and their motivations. In Proceedings of the 2011 Annual Conference on Human Factors in Computing Systems (CHI '11). ACM Press, Vancouver, BC, Canada, 3411.

[6] Gordana Apic, Matthew J Betts, and Robert B Russell. 2011. Content disputes in Wikipedia reflect geopolitical instability. PloS ONE 6, 6 (2011).

[7] Mariam Asad and Christopher A Le Dantec. 2015. Illegitimate civic participation: Supporting community activists on the ground. In Proceedings of the 18th ACM Conference on Computer Supported Cooperative Work \& Social Computing (CSCW '18). ACM, 1694-1703.

[8] Shaowen Bardzell. 2010. Feminist HCI: Taking stock and outlining an agenda for design. In Proceedings of the SIGCHI Conference on Human Factors in Computing Systems (CHI '10). ACM, 1301-1310.

[9] Shaowen Bardzell and Jeffrey Bardzell. 2011. Towards a feminist HCI methodology: Social science, feminism, and HCI. In Proceedings of the SIGCHI Conference on Human Factors in Computing Systems (CHI '11). ACM, 675-684.

[10] Yochai Benkler. 2004. Sharing nicely: On shareable goods and the emergence of sharing as a modality of economic production. The Yale Law fournal 114, 2 (2004), 273.

[11] Ivan Beschastnikh, Travis Kriplean, and David W McDonald. 2008. Wikipedian self-governance in action: Motivating the policy lens. In Proceedings of the International AAAI Conference on Web and Social Media (ICWSM '08).

[12] Patrick Biernacki and Dan Waldorf. 1981. Snowball sampling: Problems and techniques of chain referral sampling. Sociological Methods \& Research 10, 2 (1981), 141-163.

[13] Maxine Birch and Tina Miller. 2000. Inviting intimacy: The interview as therapeutic opportunity. International Journal of Social Research Methodology 3, 3 (2000), 189-202.

[14] Gro Bjerknes and Tone Bratteteig. 1995. User participation and democracy: A discussion of Scandinavian research on system development. Scandinavian fournal of Information Systems 7, 1 (1995), 73-98.

[15] Erling Björgvinsson, Pelle Ehn, and Per-Anders Hillgren. 2012. Agonistic participatory design: Working with marginalised social movements. CoDesign 8, 2-3 (2012), 127-144.

[16] Erling Bjarki Björgvinsson. 2008. Open-ended participatory design as prototypical practice. Co-Design 4, 2 (2008), 85-99.

[17] Herbert Blumer. 1986. Symbolic Interactionism: Perspective and Method. University of California Press.

[18] Tom Boellstorff, Bonnie Nardi, Celia Pearce, and Tina L Taylor. 2012. Ethnography and Virtual Worlds: A Handbook of Method. Princeton University Press.

[19] Alan Borning and Michael Muller. 2012. Next steps for value sensitive design. In Proceedings of the 2012 ACM Annual Conference on Human Factors in Computing Systems (CHI '12). ACM Press, 1125.

[20] danah boyd and Nicole Ellison. 2007. Social network sites: Definition, history, and scholarship. fournal of Computer-Mediated Communication 13, 1 (2007), 210-230.

[21] Jan Brace-Govan et al. 2004. Issues in snowball sampling: The lawyer, the model and ethics. Qualitative Research fournal 4, 1 (2004), 52.
[22] Samantha Breslin and Bimlesh Wadhwa. 2014. Exploring nuanced gender perspectives within the $\mathrm{HCI}$ community. In Proceedings of the India 2014 HCI Conference on Human Computer Interaction. ACM, 45.

[23] Kath Browne. 2005. Snowball sampling: Using social networks to research non-heterosexual women. International fournal of Social Research Methodology 8, 1 (2005), 47-60.

[24] Amy Bruckman. 2014. Research ethics and HCI. In Ways of Knowing in HCI. Springer, 449-468.

[25] Elizabeth A Buchanan and Charles M Ess. 2009. Internet research ethics and the institutional review board: Current practices and issues. Computers and Society 39, 3 (2009), 43-49.

[26] Anupam Chander and Madhavi Sunder. 2004. The romance of the public domain. California Law Review 92, 5 (2004).

[27] Kathy Charmaz. 1996. Grounded theory. In Rethinking Methods in Psychology, J.A. Smith, R. Harre, and L. Van Langenhove (Eds.). SAGE Publications, 27-49.

[28] An-Shou Cheng and Kenneth R Fleischmann. 2010. Developing a meta-inventory of human values. In Proceedings of the 73rd ASIS\&T Annual Meeting on Navigating Streams in an Information Ecosystem. American Society for Information Science, 3.

[29] Hichang Cho, MeiHui Chen, and Siyoung Chung. 2010. Testing an integrative theoretical model of knowledge-sharing behavior in the context of Wikipedia. Journal of the American Society for Information Science and Technology 61, 6 (2010), 1198-1212.

[30] Adele E Clarke. 2003. Situational analyses: Grounded theory mapping after the postmodern turn. Symbolic Interaction 26, 4 (2003), 553-576.

[31] E. Gabriella Coleman. 2012. Coding Freedom: The Ethics and Aesthetics of Hacking. Princeton University Press.

[32] Benjamin Collier and Julia Bear. 2012. Conflict, confidence, or criticism: An empirical examination of the gender gap in Wikipedia. Proceedings of the SIGCHI Conference on Computer Supported Cooperative Work (CSCW'12) (2012), 383-392.

[33] Pamela Cotterill. 1992. Interviewing women: Issues of friendship, vulnerability, and power. In Women's Studies International Forum, Vol. 15. 593-606.

[34] Susan M Cox and Michael McDonald. 2013. Ethics is for human subjects too: Participant perspectives on responsibility in health research. Social Science \& Medicine 98 (2013), 224-231.

[35] Mary L. Cummings. 2006. Integrating ethics in design through the value-sensitive design approach. Science and Engineering Ethics 12, 4 (2006), 701-715.

[36] Christopher A. Le Dantec and Carl DiSalvo. 2013. Infrastructuring and the formation of publics in participatory design. Social Studies of Science 43, 2 (2013), 241-264.

[37] Maria Puig de la Bellacasa. 2011. Matters of care in technoscience: Assembling neglected things. Social Studies of Science 41, 1 (2011), 85-106.

[38] María Puig de La Bellacasa. 2017. Matters of Care: Speculative Ethics in More than Human Worlds. University of Minnesota Press.

[39] Norman K Denzin and Yvonna S Lincoln. 2008. Strategies of Qualitative Inquiry. Vol. 2. SAGE Publications.

[40] Vinciane Despret. 2004. The body we care for: Figures of anthropozoo-genesis. Body \& Society 10, 2-3 (2004), 111-134.

[41] Virginia Dickson-Swift, Erica L James, Sandra Kippen, and Pranee Liamputtong. 2006. Blurring boundaries in qualitative health research on sensitive topics. Qualitative Health Research 16, 6 (2006), 853-871.

[42] Virginia Dickson-Swift, Erica L James, Sandra Kippen, and Pranee Liamputtong. 2009. Researching sensitive topics: Qualitative research as emotion work. Qualitative Research 9, 1 (2009), 61-79.

[43] Carl DiSalvo. 2012. Adversarial Design. The MIT Press.

[44] Carl DiSalvo, Jonathan Lukens, Thomas Lodato, Tom Jenkins, and Tanyoung Kim. 2014. Making public things: How HCI design can 
express matters of concern. In Proceedings of the SIGCHI Conference on Human Factors in Computing Systems (CHI '14). ACM, 2397-2406.

[45] Lynn Dombrowski, Ellie Harmon, and Sarah Fox. 2016. Social justiceoriented interaction design: Outlining key design strategies and commitments. In Proceedings of the 2016 ACM Conference on Designing Interactive Systems (DIS '16). ACM, 656-671.

[46] Fernando Dominguez Rubio. 2016. On the discrepancy between objects and things: An ecological approach. fournal of Material Culture 21, 1 (2016), 59-86.

[47] Stine Eckert and Linda Steiner. 2013. (Re)triggering backlash: Responses to news about Wikipedia's gender gap. Journal of Communication Inquiry 37, 4 (2013), 284-303.

[48] Pelle Ehn. 2017. Scandinavian design: On participation and skill. In Participatory Design. CRC Press, 41-77.

[49] Carolyn Ellis. 2007. Telling secrets, revealing lives: Relational ethics in research with intimate others. Qualitative Inquiry 13, 1 (2007), 3-29.

[50] Steven Epstein. 1996. Impure Science: AIDS, Activism, and the Politics of Knowledge. University of California Press.

[51] Kim Etherington. 2007. Ethical research in reflexive relationships. Qualitative inquiry 13, 5 (2007), 599-616.

[52] Frantz Fanon. 1970. Black Skin, White Masks. Grove Press.

[53] Heather Ford and Judy Wajcman. 2017. 'Anyone can edit', not everyone does: Wikipedia's infrastructure and the gender gap. Social Studies of Science 47, 4 (2017), 511-527.

[54] Michel Foucault and Paul Rabinow. 1984. What is Enlightenment? In The Foucault Reader. Vol. 43. Random House.

[55] Sarah Fox, Mariam Asad, Katherine Lo, Jill P Dimond, Lynn S Dombrowski, and Shaowen Bardzell. 2016. Exploring social justice, design, and HCI. In Proceedings of the SIGCHI Conference on Human Factors in Computing Systems (CHI '16). ACM, 3293-3300.

[56] Mark Frankel and Sanyin Siang. 1999. Ethical and legal aspects of human subjects research on the Internet. American Association for the Advancement of Science.

[57] Paulo Freire. 2018. Pedagogy of the Oppressed. Bloomsbury Publishing.

[58] Batya Friedman, Alan Borning, Janet L Davis, Brian T Gill, Peter H Kahn Jr, Travis Kriplean, and Peyina Lin. 2008. Laying the foundations for public participation and value advocacy: Interaction design for a large scale urban simulation. In Proceedings of the International Conference on Digital Government Research. 305-314.

[59] Batya Friedman, Peter H Kahn, Alan Borning, and Alina Huldtgren. 2013. Value sensitive design and information systems. In Early Engagement and New Technologies: Opening up the Laboratory. Springer, 55-95.

[60] Radhika Gajjala. 2002. An interrupted postcolonial/feminist cyberethnography: Complicity and resistance in the "cyberfield". Feminist Media Studies 2, 2 (2002).

[61] R Stuart Geiger and David Ribes. 2010. The work of sustaining order in Wikipedia: The banning of a vandal. In Proceedings of the SIGCHI Conference on Computer Supported Cooperative Work (CSCW '10). ACM, 117-126.

[62] Cathy Gere. 2017. Pain, Pleasure and the Greater Good: From the Panopticon to the Skinner Box and Beyond. The University of Chicago Press.

[63] Ruediger Glott, Philipp Schmidt, and Rishab Ghosh. 2010. Wikipedia survey: Overview of results. United Nations University, Collaborative Creativity Group (2010), 1158-1178.

[64] Scott A Golder and Michael W Macy. 2014. Digital footprints: Opportunities and challenges for online social research. Annual Review of Sociology 40 (2014), 129-152.

[65] Marilys Guillemin and Lynn Gillam. 2004. Ethics, reflexivity, and "ethically important moments" in research. Qualitative Inquiry 10, 2
(2004), 261-280.

[66] Aaron Halfaker, R. Stuart Geiger, Jonathan T. Morgan, and John Riedl. 2013. The rise and decline of an open collaboration system: How Wikipedia's reaction to popularity is causing its decline. American Behavioral Scientist 57, 5 (May 2013), 664-688.

[67] Aaron Halfaker, Aniket Kittur, and John Riedl. 2011. Don't bite the newbies: How reverts affect the quantity and quality of Wikipedia work. In Proceedings of the 7th International Symposium on Wikis and Open Collaboration (WikiSym '11). ACM, 163-172.

[68] Maurice Hamington et al. 2004. Embodied Care: Fane Addams, Maurice Merleau-Ponty, and Feminist Ethics. University of Illinois Press.

[69] Martyn Hammersley and Paul Atkinson. 2007. Ethnography: Principles in Practice. Routledge.

[70] Donna Haraway. 1988. Situated knowledges: The science question in feminism and the privilege of partial perspective. Feminist Studies 14, 3 (1988), 575-599.

[71] Donna Haraway. 2007. When Species Meet. University of Minnesota Press.

[72] Sandra Harding. 1992. Rethinking standpoint epistemology: What is "strong objectivity?". The Centennial Review 36, 3 (1992), 437-470.

[73] Sandra G Harding. 1986. The Science Question in Feminism. Cornell University Press.

[74] Eszter Hargittai and Aaron Shaw. 2015. Mind the skills gap: The role of Internet know-how and gender in differentiated contributions to Wikipedia. Information, Communication \& Society 18, 4 (2015), 424-442.

[75] Kieran Healy and Alan Schussman. 2003. The ecology of open-source software development. Technical Report. Department of Sociology. University of Arizona.

[76] Charlotte Hess and Elinor Ostrim. 2008. Understanding knowledge as a commons: From theory to practice. Fournal of the American Society for Information Science and Technology 59, 2 (2008), 333-334.

[77] Benjamin Mako Hill and Aaron Shaw. 2013. The Wikipedia gender gap revisited: Characterizing survey response bias with propensity score estimation. PLoS ONE 8, 6 (June 2013).

[78] Christine Hine. 2000. Virtual Ethnography. SAGE Publications.

[79] Douglas R Holmes and George E Marcus. 2008. Collaboration today and the re-imagination of the classic scene of fieldwork encounter. Collaborative Anthropologies 1, 1 (2008), 81-101.

[80] Bell Hooks. 2014. Teaching To Transgress. Routledge.

[81] James M Hudson and Amy Bruckman. 2004. "Go away": Participant objections to being studied and the ethics of chatroom research. The Information Society 20, 2 (2004), 127-139.

[82] Lilly Irani, Janet Vertesi, Paul Dourish, Kavita Philip, and Rebecca E Grinter. 2010. Postcolonial computing: A lens on design and development. In Proceedings of the SIGCHI Conference on Human Factors in Computing Systems (CHI '10). ACM, 1311-1320.

[83] Lilly C Irani and M Silberman. 2016. Stories we tell about labor: Turkopticon and the trouble with design. In Proceedings of the SIGCHI Conference on Human Factors in Computing Systems (CHI '16). ACM, 4573-4586.

[84] Steven J Jackson. 2014. Rethinking repair. In Media Technologies: Essays on Communication, Materiality, and Society. MIT Press, 221239.

[85] Nassim JafariNaimi, Lisa Nathan, and Ian Hargraves. 2015. Values as hypotheses: Design, inquiry, and the service of values. Design Issues 31, 4 (2015), 91-104.

[86] Dariusz Jemielniak. 2014. Common Knowledge?: An Ethnography of Wikipedia. Stanford University Press.

[87] Andreas M Kaplan and Michael Haenlein. 2010. Users of the world, unite! The challenges and opportunities of social media. Business 
Horizons 53, 1 (2010), 59-68.

[88] Orit Karnieli-Miller, Roni Strier, and Liat Pessach. 2009. Power relations in qualitative research. Qualitative Health Research 19, 2 (2009), 279-289.

[89] Christopher M. Kelty. 2008. Two Bits: The Cultural Significance of Free Software. Duke University Press.

[90] Finn Kensing and Jeanette Blomberg. 1998. Participatory design: Issues and concerns. Computer Supported Cooperative Work (CSCW '98) 7, 3-4 (1998), 167-185.

[91] Eva Feder Kittay and Ellen K Feder. 2003. The Subject of Care: Feminist Perspectives on Dependency. Rowman \& Littlefield.

[92] Aniket Kittur, Ed H Chi, and Bongwon Suh. 2008. Crowdsourcing user studies with Mechanical Turk. In Proceedings of the SIGCHI Conference on Human Factors in Computing Systems (CHI '08). ACM, 453-456.

[93] Aniket Kittur, Bongwon Suh, Bryan A. Pendleton, and Ed H. Chi. 2007. He says, she says: Conflict and coordination in Wikipedia. In Proceedings of the SIGCHI Conference on Human Factors in Computing Systems (CHI '07). ACM Press, San Jose, California, USA, 453.

[94] Shyong (Tony) K. Lam, Anuradha Uduwage, Zhenhua Dong, Shilad Sen, David R. Musicant, Loren Terveen, and John Riedl. 2011. WP:clubhouse?: An exploration of Wikipedia's gender imbalance. In Proceedings of the 7th International Symposium on Wikis and Open Collaboration (WikiSym '11). ACM Press, 1.

[95] Joanna Latimer and Maria Puig de la Bellacasa. 2013. Re-thinking the ethical: Everyday shifts of care in biogerontology. Ethics, Law and Society 5 (2013), 153-174.

[96] Bruno Latour. 2008. A cautious prometheus?: A few steps toward a philosophy of design (with special attention to Peter Sloterdijk). In Proceedings of the 2008 annual international conference of the Design History Society. 2-10.

[97] Bruno Latour and Steve Woolgar. 2013. Laboratory life: The Construction of Scientific facts. Princeton University Press.

[98] Christopher A Le Dantec, Erika Shehan Poole, and Susan P Wyche. 2009. Values as lived experience: Evolving value sensitive design in support of value discovery. In Proceedings of the SIGCHI Conference on Human Factors in Computing Systems (CHI '09). ACM, 1141-1150.

[99] Norman K Denzin Yvonna S Lincoln, Yvonna S Lincoln, et al. 2005. The Sage Handbook of Qualitative Research. SAGE Publications.

[100] Yvonna S. Lincoln and Egon G. Guba. 1985. Naturalistic Inquiry. SAGE Publications.

[101] Aryn Martin, Natasha Myers, and Ana Viseu. 2015. The politics of care in technoscience. Social Studies of Science 45, 5 (2015), 625-641.

[102] Adrienne Massanari. 2017. \#Gamergate and The Fappening: How Reddit's algorithm, governance, and culture support toxic technocultures. New Media \& Society 19, 3 (2017), 329-346.

[103] Annemarie Mol. 2008. The Logic of Care: Health and the Problem of Patient Choice. Routledge.

[104] Wendy Moncur. 2013. The emotional wellbeing of researchers: Considerations for practice. Proceedings of the SIGCHI Conference on Human Factors in Computing Systems (CHI '13) (2013), 8.

[105] Jonathan T Morgan, Siko Bouterse, Heather Walls, and Sarah Stierch. 2013. Tea and sympathy: Crafting positive new user experiences on Wikipedia. In Proceedings of the SIGCHI Conference on Computer Supported Cooperative Work (CSCW'13). ACM, 839-848.

[106] Norma Morris and Brian Bàlmer. 2006. Volunteer human subjects' understandings of their participation in a biomedical research experiment. Social Science \& Medicine 62, 4 (2006), 998-1008.

[107] Torill Elvira Mortensen. 2018. Anger, fear, and games: The long event of \#GamerGate. Games and Culture 13, 8 (2018), 787-806.

[108] Michael J Muller and Allison Druin. 2003. Participatory design: The third space in HCI. In Human-Computer Interaction: Development Process.
[109] Ruth Müller and Martha Kenney. 2014. Agential conversations: Interviewing postdoctoral life scientists and the politics of mundane research practices. Science as Culture 23, 4 (2014), 537-559.

[110] Cosmin Munteanu, Heather Molyneaux, Wendy Moncur, Mario Romero, Susan O’Donnell, and John Vines. 2015. Situational ethics: Re-thinking approaches to formal ethics requirements for humancomputer interaction. In Proceedings of the SIGCHI Conference on Human Factors in Computing Systems (CHI '15). ACM Press, Seoul, Republic of Korea, 105-114.

[111] Keith M. Murphy. 2019. Swedish Design: An Ethnography. Cornell University Press.

[112] Michelle Murphy. 2015. Unsettling care: Troubling transnational itineraries of care in feminist health practices. Social Studies of Science 45, 5 (2015), 717-737.

[113] Lisa P. Nathan, Anja Thieme, Deborah Tatar, and Stacy Branham. 2016. Disruptions, dilemmas and paradoxes: Ethical matter(s) in design research. Interacting with Computers (2016), 1-9.

[114] Safiya Umoja Noble. 2018. Algorithms of Oppression: How Search Engines Reinforce Racism. NYU Press.

[115] Wikimedia Foundation Board of Trustees. 2016. Statement on healthy community culture, inclusivity, and safe spaces. Retrieved December 14, 2018 from https://web.archive.org/web/20190107195418/https: //meta.wikimedia.org/wiki/Wikimedia_Foundation_Board_ noticeboard/November_2016_-_Statement_on_Healthy_ Community_Culture,_Inclusivity,_and_Safe_Spaces

[116] Julian E Orr. 1990. Sharing knowledge, celebrating identity: Community memory in a service culture. In Collective Remembering. SAGE Publications.

[117] Sherry B Ortner. 1984. Theory in anthropology since the sixties. Comparative Studies in Society and History 26, 1 (1984), 126-166.

[118] Judith Phillips. 2007. Care. Polity.

[119] Joseph Reagle and Lauren Rhue. 2011. Gender bias in Wikipedia and Britannica. International fournal of Communication 5 (2011), 21.

[120] Joseph Michael Reagle. 2010. Good Faith Collaboration: The Culture of Wikipedia. MIT Press.

[121] Annelise Riles. 2015. From comparison to collaboration: Experiments with a new scholarly and political reform. Law \& Contemporary Problems 78 (2015), 147.

[122] Jennifer A Rode. 2011. Reflexivity in digital anthropology. In Proceedings of the SIGCHI Conference on Human Factors in Computing Systems (CHI '11). ACM, 123-132.

[123] Jennifer A Rode. 2011. A theoretical agenda for feminist HCI. Interacting with Computers 23, 5 (2011), 393-400.

[124] Anita Sarkeesian. 2012. Harassment via wikipedia vandalism. Retrieved November 19, 2018 from https://feministfrequency.com/2012/ 06/10/harassment-and-misogyny-via-wikipedia/

[125] Ralph Schroeder and Linnet Taylor. 2015. Big data and Wikipedia research: Social science knowledge across disciplinary divides. Information, Communication \& Society 18, 9 (2015), 1039-1056.

[126] Douglas Schuler and Aki Namioka. 1993. Participatory Design: Principles and Practices. CRC Press.

[127] Carolyn B Seaman. 1999. Qualitative methods in empirical studies of software engineering. IEEE Transactions on Software Engineering 4 (1999), 557-572.

[128] Phoebe Sengers, Kirsten Boehner, Shay David, and Joseph 'Jofish' Kaye. 2005. Reflective design. In Proceedings of the 4th Decennial Conference on Critical Computing between Sense and Sensibility (CC '05). ACM Press, Aarhus, Denmark, 49.

[129] Irina Shklovski and Janet Vertesi. 2012. "Un-Googling": Research technologies, communities at risk and the ethics of user studies in HCI. In Proceedings of the BCS HCI Workshop on HCI Research in Sensitive Contexts: Ethical Considerations. 
[130] Dorothy E Smith. 1990. The Conceptual Practices of Power: A Feminist Sociology of Knowledge. University of Toronto Press.

[131] Marilyn Strathern. 1985. Dislodging a world view: Challenge and counter-challenge in the relationship between feminism and anthropology. Australian Feminist Studies 1, 1 (1985), 1-25.

[132] Anselm Strauss. 1982. Social worlds and legitimation processes. Studies in Symbolic Interaction 4, 17 (1982).

[133] Lucy Suchman. 2002. Located accountabilities in technology production. Scandinavian fournal of Information Systems 14, 2 (2002), 7.

[134] Lucy A Suchman. 1987. Plans and Situated Actions: The Problem of Human-Machine Communication. Cambridge University Press.

[135] Alex Taylor. 2011. Out there. Proceedings of the SIGCHI Conference on Human Factors in Computing Systems (CHI '11) (2011), 10.

[136] John Torous and Camille Nebeker. 2017. Navigating ethics in the digital age: Introducing Connected and Open Research Ethics (CORE), a tool for researchers and institutional review boards. Fournal of Medical Internet Research 19, 2 (2017).

[137] Joan C Tronto. 1993. Moral Boundaries: A Political Argument for an Ethic of Care. Psychology Press.

[138] Jose van Dijck. 2013. The Culture of Connectivity: A Critical History of Social Media. Oxford University Press.

[139] Fernanda B. Viégas, Martin Wattenberg, and Kushal Dave. 2004. Studying cooperation and conflict between authors with history flow visualizations. In Proceedings of the SIGCHI Conference on Human
Factors in Computing Systems (CHI '04). ACM, 575-582.

[140] Kamala Visweswaran. 1994. Fictions of Feminist Ethnography. University of Minnesota Press.

[141] Kamala Visweswaran. 1997. Histories of feminist ethnography. Annual Review of Anthropology 26, 1 (1997), 591-621.

[142] Martin Wattenberg, Fernanda B Viégas, and Katherine Hollenbach. 2007. Visualizing activity on Wikipedia with chromograms. In IFIP Conference on Human-Computer Interaction. Springer, 272-287.

[143] Etienne Wenger. 1999. Communities of Practice: Learning, Meaning, and Identity. Cambridge University Press.

[144] Sarah Whatmore. 1997. Dissecting the autonomous self: Hybrid cartographies for a relational ethics. Environment and Planning: Society and Space 15, 1 (Feb. 1997), 37-53.

[145] Elena Ariel Windsong. 2018. Incorporating intersectionality into research design: An example using qualitative interviews. International fournal of Social Research Methodology 21, 2 (March 2018), 135-147.

[146] L Mun Wong. 1998. The ethics of rapport: Institutional safeguards, resistance, and betrayal. Qualitative Inquiry 4, 2 (1998), 178-199.

[147] Fei Wu and Daniel S Weld. 2007. Autonomously semantifying wikipedia. In Proceedings of the ACM Conference on Conference on Information and Knowledge Management. ACM, 41-50.

[148] Taha Yasseri, Robert Sumi, András Rung, András Kornai, and János Kertész. 2012. Dynamics of conflicts in Wikipedia. PloS ONE 7, 6 (2012). 